\title{
Well-Treated GDM doesn't Worsen Obstetric Outcomes in Obese Women
}

\author{
D’Anna $\mathrm{R}^{* 1}$, Ruggeri $\mathrm{C}^{1}$, Corrado $\mathrm{F}^{1}$ and Di Benedetto $\mathrm{A}^{2}$ \\ ${ }^{1}$ Department of Human Pathology, University of Messina, Italy \\ ${ }^{2}$ Department of Internal Medicine, University of Messina, Italy
}

*Corresponding author: Rosario D’Anna, Department of Human Pathology, University of Messina, Italy Viale Italian. 2, 98124 Messina, Italy

To Cite This Article: D'Anna R, Ruggeri C, Corrado F, Di Benedetto A. Well-Treated GDM doesn't Worsen Obstetric Outcomes in Obese Women. 2020 - 9(2). AJBSR.MS.ID.001361. DOI: 10.34297/AJBSR.2020.09.001361.

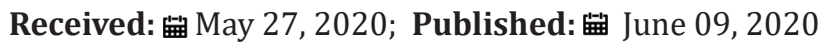

\begin{abstract}
Aim: To compare obstetric outcomes in obese women with and without Gestational Diabetes Mellitus (GDM)

Methods: This is a single-centre retrospective study carried out on the obese pregnant women's charts in a 5-year period. After an Oral Glucose Tolerance Test for GDM screening, obese women who developed GDM were carefully treated by the same diabetologist. The outcome measures considered were caesarean section rate in emergency, pre-term birth, gestational hypertension and large for gestational age babies.

Results: Among 6586 women who delivered in the period considered, 425 (6.4\%) were obese; of whom 116 (27.3\%) developed GDM while 309 $(72.3 \%)$ did not. In the same period, other 550 non obese women with GDM were considered as control group. Between obese groups, there were no differences in all the outcome measures considered. Instead, in relation to gestational hypertension a difference statistically significant was shown between all obese women and non-obese women with GDM.
\end{abstract}

Conclusion: This study has shown that in well-treated GDM obese women the obstetric outcomes were not significantly different compared to pregnant obese women without GDM, suggesting that the main problem for these women is obesity, which it seems more serious than GDM alone.

Keywords: GDM, Obese pregnant women, Obstetric outcomes

\section{Introduction}

The prevalence of obesity is increasing worldwide contributing a progressive increase of gestational diabetes mellitus (GDM) rate. In obese women without GDM but also in non-obese women with GDM an increased risk for macrosomia, hypertensive disorders and primary caesarean section has been reported [1]. Moreover, the combination of GDM and pre-pregnancy obesity, commonly named diabesity, showed a greater risk of adverse pregnancy outcomes than either GDM or obesity alone [1]. In particular, untreated obese pregnant women who were enrolled and categorized as diabetic showed several adverse pregnancy outcomes [1]. Similar results were reported in a large register-based, multicenter Finnish study [2]. On the contrary, some reports have demonstrated that diabesity not necessarily it means a worse obstetric outcome compared to obesity without GDM $[3,4]$. In fact, in a Chinese population, an important outcome as macrosomia was four times greater in obese women than in lean women with GDM [3]. In another, more recent study, pre-pregnancy obesity had a rate of preeclampsia and preterm birth higher than the diabesity group [4]. In this study, we wanted to verify in our population whether a well-treated GDM in obese women may further worsen pregnancy outcomes compared to obese women without GDM.

\section{Methods}

This is a single-centre retrospective study carried out on clinical records of the consecutive obese pregnant women who delivered in the University Hospital of Messina (Italy) from January 2015 until December 2019 . Were included 425 obese women (BMI $\geq 30 \mathrm{~kg} / \mathrm{m}^{2}$ ) with a single pregnancy, who performed an oral Glucose Tolerance Test (OGTT) at 16-18 weeks gestation and then, if negative, a further OGTT at 24-28 weeks gestation, in accordance with Italian Guidelines [5]. Threshold values were $\geq 92 \mathrm{mg} / \mathrm{dL}$ fasting, $\geq 180$ $\mathrm{mg} / \mathrm{dL}$ at 1 hour after load, and $\geq 153 \mathrm{mg} / \mathrm{dL}$ at 2 hours after 
load. One of the 3 values that exceeds or equals the threshold was diagnostic of GDM. Each woman who met GDM criteria received a specific diet and/or insulin when required, according to American Diabetes Association guidelines [6]. Furthermore, they were carefully checked monthly. The other non-obese women with GDM diagnosed in the same period, were included as controls. The measured outcomes have been the rate of caesarean section in emergency, gestational hypertension, pre-term birth and Large for Gestational Age (LGA) babies. Gestational hypertension was defined as blood pressure $\geq 140 / 90 \mathrm{~mm}$ Hg that was measured twice, at least 6 hours apart, after 20 weeks of gestation ; preterm birth was defined as delivery at $<259$ days since the last menstrual period; LGA babies were evaluated according to Italian Charts on neonatal anthropometric measures, as $\geq 90$ th percentile [7].

\section{Statistics}

The numeric data are expressed as mean \pm standard deviation, and the categorical variables are expressed as count and percentage. The Student $\mathrm{t}$ test, Mann-Whitney test, and chi-square test were applied where appropriate. Statistical analysis was performed with
IBM SPSS Statistics for Windows (version 17; IBM Corporation, Armonk, NY). A value of <.05 was considered statistically significant.

\section{Results}

In our Hospital, in the 5 -year period considered, 6586 deliveries with 666 GDM cases (10.1\%) occurred. Of 425 (6.4\%) obese women enrolled, 116 (27.3\%) developed GDM while 309 (72.7\%) did not. All the non-obese women affected by GDM (550) who delivered in the same period, were considered as an additional control group, with a total sample of 975 women enrolled, and a GDM rate of 14.8\%. Maternal and neonatal characteristics (Table 1) evidenced some statistically significant differences between obese groups. In particular, maternal age and pre-pregnancy BMI were significantly higher in the diabesity group, whereas gestational weight gain and gestational age at delivery were significantly higher in the obese group without GDM. In relation to outcome measures (Table 2), there were no statistical difference between the 2 groups of obese women. Instead, a difference statistically significant between obese without GDM and non-obese with GDM group was shown for gestational hypertension and pre-term birth.

\begin{tabular}{|c|c|c|c|}
\hline CHARACTERISTICS & OBESITY n. 309 & DIABESITY n. 116 & $\mathbf{P}$ \\
\hline MATERNAL AGE (y) & $31.3 \pm 5.5$ & $33.3 \pm 4.9$ & 0.0006 \\
\hline NULLIPAROUS (n) & $125(40.4 \%)$ & $51(43.9 \%)$ & 0.58 \\
\hline PRE- BMI $\left(\mathrm{Kg} / \mathrm{m}^{2}\right)$ & $34.2 \pm 4.1$ & $35.1 \pm 4.2$ & 0.04 \\
\hline GWG (Kg) & $9.0 \pm 5.4$ & $7.8 \pm 5.7$ & 0.04 \\
\hline GA AT DELIVERY (d) & $273 \pm 12$ & $267 \pm 12$ & $<0.0001$ \\
\hline BIRTH WEIGHT & $3280 \pm 480$ & $3260 \pm 452$ & 0.69 \\
\hline
\end{tabular}

\begin{tabular}{|c|c|c|c|}
\hline Table 2: Comparison of clinical outcomes among groups \\
\hline OUTCOMES & OBESE NO GDM (n. 309) & DIABESITY (n. 116) & GDM NON OBESE (n. 550) \\
\hline & & & $20(17.2 \%)$ \\
\hline CS in emergency & $37(12 \%)$ & $33(28.4 \%)$ & $50(14.5 \%)$ \\
\hline Gestational Hypertension & $71(23 \%)^{*}$ & $10(8,6 \%)$ & $56(10.2 \%)^{*}$ \\
\hline Pre-term birth & $18(5,8 \%)^{* *}$ & $5(4.3 \%)$ & $45(8.2 \%)$ \\
\hline LGA & $19(6.2 \%)$ & & \\
\hline \multicolumn{2}{|c|}{ CS = Caesarean Section; LGA $=$ Large for Gestational Age; ${ }^{*} \mathrm{P}<0.0001 ;{ }^{* *} \mathrm{p}=0.02$} \\
\hline
\end{tabular}

\section{Discussion}

In our setting, it seems that a well-treated GDM does not modify the risk related to a pregnancy in an obese woman, even if the comparison of some general characteristics of the 2 obese groups revealed differences statistically significant. In particular, two independent risk factors for worse pregnancy outcomes, such as maternal age and pre-gestational BMI were higher in the diabesity group, indicating that this latter group should be at major risk for adverse pregnancy outcomes. Instead, there were no differences between these groups and the explanation might be a more accurate management of the pregnancy, when complicated by GDM which also benefits the obesity condition. In fact, a significant difference in gestational weight gain was shown advantaging just the obese group with GDM. However, this study has some weaknesses and limitations. First of all, the number of the women enrolled, but also the retrospective nature of the study. Furthermore, being a single centre, this sample of women might be not representative of the whole country. On the other hand, the homogenous therapeutic management performed by the same diabetologist, might be a point of strength. A recent similar study [8] considered 3 groups like ours, obtaining similar results on obstetric outcomes such as hypertensive syndromes and LGA babies. In particular, the rate 
of hypertensive syndromes was statistically significant higher in obese without GDM women compared to non-obese women with GDM. The same result was obtained also for macrosomia (birth weight $\geq 4 \mathrm{~kg}$ ) with respect to all women with GDM, also to those obese. The Authors suggested that this latter result was related to the fact that women with GDM and LGA babies were induced between 38-39 weeks gestation. It's well known that obesity and GDM are independent risk factors for adverse pregnancy outcomes, and that diabesity may cause a higher rate of macrosomia compared to obese women without GDM [2]. But the HAPO study [1] told us that a poor treatment of GDM was principally responsible of worse pregnancy outcomes.

In conclusion, this study has shown that in well-treated GDM obese women, the obstetric outcomes were not significantly different compared to pregnant obese women without GDM, suggesting that the main problem for these women is obesity, which it seems more serious than GDM alone.

\section{References}

1. Catalano PM, McIntire HD, Cruickshank JK, McCance DR, Dyer AR, et al. (2012) The Hyperglycemia and Adverse Pregnancy Outcome study: Associations of GDM and obesity with pregnancy outcomes. Diabetes Care 35(4): 780-786
2. Ijas H, Koivunen S, Raudaskoski T, Kajantie E, Gissler M, et al. (2019) Independent and concomitant association of gestational diabetes and maternal obesity to perinatal outcome: a register-based study. Plos One 14(8): e0221549.

3. Wang LF, Wang HJ, Ao D, Wang Y, Yang HX, et al. (2015) Influence of prepregnancy obesity on the development of macrosomia and large for gestational age in women with or without gestational diabetes mellitus in Chinese population. J Perinatol 35(12): 985-990.

4. Blickstein I, Doyev R, Trojner Bregar A, Brzan Simenc G, Verdenik I, et al. (2018) The effect of gestational diabetes, pre-gravid maternal obesity, and their combination (diabesity) on outcomes of singleton gestations. J Matern Fetal Neonatal Med 31(5): 640-643.

5. "Istituto Superiore di Sanità", Lineeguida per la gravidanzafisiologica.

6. American Diabetes Association (2011) Standards of medical care in diabetes-2011. Diabetes Care 34(suppl 1): s11-s61.

7. Bertino E, Spada E, Occhi L, Coscia A, Giuliani F, et al. (2010) Neonatal anthropometric charts: The Italian neonatal study compared with other European studies. J Pediatr Gastroenterol Nutr 51(3): 353-361.

8. Huet J, Beucher G, Rod A, Morello R, Dreyfus M (2018) Joint impact of gestational diabetes and obesity on perinatal outcomes. J Gynecol Obstet Hum Reprod 47(9): 469-476. 